1 Hacettepe Journal of Mathematics and Statistics

holume 46 (1) (2017), $67-76$

\title{
Characterizations of quasi-metric completeness in terms of Kannan-type fixed point theorems
}

\author{
Dedicated to the memory of Professor Lawrence M. Brown \\ Carmen Alegre*, Hacer Dağ ${ }^{\dagger}$, Salvador Romaguera ${ }^{\ddagger}$ and Pedro Tirado ${ }^{\S}$
}

\begin{abstract}
We obtain quasi-metric versions of Kannan's fixed point theorem for self-mappings and multivalued mappings, respectively, which are used to deduce characterizations of $d$-sequentially complete and of left $\mathrm{K}$ sequentially complete quasi-metric spaces, respectively.
\end{abstract}

Keywords: Quasi-metric space, complete, Kannan mapping, fixed point. 2000 AMS Classification: 54H25, 54E50, 47H10.

\section{Introduction and preliminaries}

Since Hu proved in [10] that a metric space $(X, d)$ is complete if and only if for any closed subspace $C$ of $(X, d)$, every Banach contraction on $C$ has fixed point, several authors have investigated the problem of characterizing the metric completeness with the help of fixed point theorems (see e.g. [13, 18, 25, 26, 27, 28]). Next we recall those characterizations which will be related with our approach.

\footnotetext{
*Instituto Universitario de Matemática Pura y Aplicada, Universitat Politècnica de València, 46022 Valencia, Spain

${ }^{\dagger}$ Departamento de Matemática Aplicada, Universitat Politècnica de València, 46022 Valencia, Spain

Email : hada@doctor.upv.es

$\ddagger$ Instituto Universitario de Matemática Pura y Aplicada, Universitat Politècnica de València, 46022 Valencia, Spain

Departamento de Matemática Aplicada, Universitat Politècnica de València, 46022 Valencia, Spain

Email : sromague@mat.upv.es, Corresponding author.

§Instituto Universitario de Matemática Pura y Aplicada, Universitat Politècnica de València, 46022 Valencia, Spain

Email : pedtipe@mat.upv.es

Doi : 10.15672/HJMS.2016.395
} 
Caristi proved in [6] the following important generalization of the Banach contraction principle.

1.1. Theorem (see [6]). Let $(X, d)$ be a complete metric space. If $T$ is a selfmapping of $X$ such that there is a lower semicontinuous function $\varphi: X \rightarrow[0, \infty)$ satisfying

$$
d(x, T x) \leq \varphi(x)-\varphi(T x),
$$

for all $x \in X$, then $T$ has a fixed point.

A self-mapping $T$ on a metric space $(X, d)$ for which there is a lower semicontinuous function $\varphi: X \rightarrow[0, \infty)$ satisfying condition (1.1) for all $x \in X$, is called a Caristi mapping on $(X, d)$.

Kirk proved in [13] that Caristi's fixed point theorem allows to characterize the metric completeness as follows.

1.2. Theorem (see [13]). A metric space $(X, d)$ is complete if and only if every Caristi mapping on $(X, d)$ has a fixed point.

Almost simultaneously, Subrahmanyam [26] showed that the well-known Kannan fixed point theorem (see Theorem 1.3 below) also allows to characterize the metric completeness.

1.3. Theorem (see [11]). Let $(X, d)$ be a complete metric space. If $T$ is a self-mapping of $X$ such that there is a constant $c \in[0,1 / 2)$ satisfying

$$
d(T x, T y) \leq c(d(x, T x)+d(y, T y))
$$

for all $x, y \in X$, then $T$ has a unique fixed point.

The above result suggests the following well-established notion: A self-mapping $T$ of a metric space $(X, d)$ is said to be a Kannan mapping on $(X, d)$ if there exists a constant $c \in[0,1 / 2)$ for which condition (1.2) is satisfied for all $x, y \in X$.

Then, Subrahmanyam proved the following.

1.4. Theorem (see [26]). A metric space $(X, d)$ is complete if and only if every Kannan mapping on $(X, d)$ has a fixed point.

On the other hand, and motivated in part by the fact that quasi-metric spaces provide suitable frameworks in several areas of asymmetric functional analysis, domain theory, and complexity analysis of algorithms defined by recurrence equations (see [8] and its bibliography, [4, 20, 21, 23, 24] etc.), the development of the fixed point theory for theses spaces is receiving a significant boost (see e.g. $[1,2,3,5,7,9,12,15,16,17])$. In this setting, the problem of characterizing quasi-metric completeness via fixed point theorems arises in a natural way. This problem has an extra appeal due to the existence of several different notions of quasi-metric completeness in the literature, so it seems reasonable to expect the existence of interesting differences with respect to the classical metric setting. In this 
paper we show that this is the case. Indeed, Romaguera and Tirado [22] extended Kirk's characterization (Theorem 1.2) to the realm of Smtyh complete quasi-metric spaces, while here we discuss the problem of characterizing the quasi-metric completeness by using appropriate versions of Kannan's fixed point theorem. In this fashion, we shall obtain characterizations of $d$-sequentially complete and of left $\mathrm{K}$-sequentially complete quasi-metric spaces, respectively.

We conclude this section by recalling some pertinent notions and properties on quasi-metric spaces which will be useful later on. (By $\mathbb{N}$ we will denote the set of all positive integer numbers.)

Following the modern terminology (see [8]), a quasi-metric on a set $X$ is a function $d: X \times X \rightarrow[0, \infty)$ such that for all $x, y, z \in X:$

(i) $x=y \Leftrightarrow d(x, y)=d(y, x)=0$, and

(ii) $d(x, z) \leq d(x, y)+d(y, z)$.

A quasi-metric space is a pair $(X, d)$ such that $X$ is a set and $d$ is a quasi-metric on $X$.

Given a quasi-metric $d$ on a set $X$ the function $d^{s}$ defined on $X \times X$ by $d^{s}(x, y)=$ $\max \{d(x, y), d(y, x)\}$ for all $x, y \in X$, is a metric on $X$.

Each quasi-metric $d$ on $X$ induces a $T_{0}$ topology $\tau_{d}$ on $X$ which has as a base the family of open balls $\left\{B_{d}(x, r): x \in X, \varepsilon>0\right\}$, where $B_{d}(x, \varepsilon)=\{y \in X$ : $d(x, y)<\varepsilon\}$ for all $x \in X$ and $\varepsilon>0$.

If $\tau_{d}$ is a $T_{1}$ topology on $X$, we say that $d$ is a $T_{1}$ quasi-metric on $X$.

A sequence $\left(x_{n}\right)_{n \in \mathbb{N}}$ in a quasi-metric space $(X, d)$ is called left K-Cauchy [19] if for each $\varepsilon>0$ there exists $n_{\varepsilon} \in \mathbb{N}$ such that $d\left(x_{n}, x_{m}\right)<\varepsilon$ whenever $n_{\varepsilon} \leq n \leq m$.

A quasi-metric space $(X, d)$ is called left K-sequentially complete (resp. $d$ sequentially complete) $[8,19]$ if every left K-Cauchy sequence in $(X, d)$ (resp. every Cauchy sequence in the metric space $\left(X, d^{s}\right)$ ) converges for the topology $\tau_{d}$, and it is called Smyth complete (see e.g. [14, 22, 23]) if every left K-Cauchy sequence in $(X, d)$ converges for the topology $\tau_{d^{s}}$.

The following implications are obvious for a quasi-metric space $(X, d)$ :

Smyth complete $\Rightarrow$ left K-sequentially complete $\Rightarrow d$-sequentially complete.

The converse implications do not hold in general. The following known examples illustrate this fact.

1.5. Example. Let $X=\mathbb{N} \cup\{0\}$ and let $d$ be the $T_{1}$ quasi-metric on $X$ given by $d(x, x)=0$ for all $x \in X, d(0, x)=1 / x$ for all $n \in \mathbb{N}$, and $d(x, y)=1$ otherwise. Then $(X, d)$ is clearly left $\mathrm{K}$-sequentially complete (note that $\tau_{d}$ is a compact topology on $X$ ), but it is not Smyth complete because the sequence $(n)_{n \in \mathbb{N}}$ is left K-Cauchy sequence but does not converge for $\tau_{d^{s}}$.

1.6. Example. Let $\mathbb{R}$ be the set of all real numbers and let $d$ be the $T_{1}$ quasimetric on $\mathbb{R}$ given by $d(x, y)=y-x$ if $x \leq y$, and $d(x, y)=1$ if $x>y$. Then $(\mathbb{R}, d)$ is $d$-sequentially complete because the Cauchy sequences in the metric space $\left(\mathbb{R}, d^{s}\right)$ are eventually constant. However, it is not left K-sequentially complete because the sequence $(-1 / n)_{n \in \mathbb{N}}$ is left K-Cauchy but does not converge for $\tau_{d^{s}}$. Observe that $\tau_{d}$ is the well-known Sorgenfrey topology on $\mathbb{R}$. 


\section{The results}

In [22], Smyth complete quasi-metric spaces were characterized by means of an appropriate quasi-metric version of Caristi's fixed point theorem.

According to [22], a self-mapping $T$ of a quasi-metric space $(X, d)$ is said to be a $d^{s}$-Caristi mapping on $(X, d)$ if there exists a function $\varphi: X \rightarrow[0, \infty)$ which is lower semicontinuous for $\tau_{d^{s}}$ and satisfies $d(x, T x) \leq \varphi(x)-\varphi(T x)$, for all $x \in X$.

Then it was proved the following.

2.1. Theorem (see [22]). A quasi-metric space $(X, d)$ is Smyth complete if and only if every $d^{s}$-Caristi mapping on $(X, d)$ has a fixed point.

In the sequel we shall prove that, however, quasi-metric versions of Kannan's fixed point theorem for self-mappings and multivalued mappings characterize $d$ sequential completeness and left K-sequential completeness, respectively.

2.2. Definition. Let $(X, d)$ be a quasi-metric space. By a $d$-Kannan mapping on $(X, d)$ we mean a self-mapping $T$ of $X$ such that there exists a constant $c \in[0,1 / 2)$ satisfying

$$
d(T x, T y) \leq c(d(x, T x)+d(y, T y)),
$$

for all $x, y \in X$.

2.3. Lemma. Let $T$ be a $d$-Kannan mapping on a quasi-metric space $(X, d)$ with constant $c \in[0,1 / 2)$. Then:

(a) $d^{s}(T x, T y) \leq c(d(x, T x)+d(y, T y))$, for all $x, y \in X$.

(b) $T$ is a Kannan mapping on the metric space $\left(X, d^{s}\right)$.

(c) For any $x_{0} \in X$, the sequence $\left(T^{n} x_{0}\right)_{n \in \mathbb{N}}$ is a Cauchy sequence in the metric space $\left(X, d^{s}\right)$.

Proof. (a) Given $x, y \in X$ we have

$$
d(T x, T y) \leq c(d(x, T x)+d(y, T y)) \quad \text { and } \quad d(T y, T x) \leq c(d(y, T y)+d(x, T x)),
$$

so

$$
d^{s}(T x, T y) \leq c(d(x, T x)+d(y, T y)) \leq c(d(x, T x)+d(y, T y)) .
$$

(b) Since $d(x, T x) \leq d^{s}(x, T x)$ and $d(y, T y) \leq d^{s}(y, T y)$ for all $x, y \in X$, it follows from assertion (a) that $T$ is a Kannan mapping on $\left(X, d^{s}\right)$, with constant $c$.

(c) Since, by (b), $T$ is a Kannan mapping for the metric space $\left(X, d^{s}\right)$, the classical proof of Kannan's fixed point theorem [11] shows that for any $x_{0} \in X$, $\left(T^{n} x_{0}\right)_{n \in \mathbb{N}}$ is a Cauchy sequence in the metric space $\left(X, d^{s}\right)$.

Related to Lemma 2.3 (b) we give an example of a self-mapping of a quasimetric space $(X, d)$ which is a Kannan mapping on $\left(X, d^{s}\right)$ but not a $d$-Kannan 
mapping.

2.4. Example. Let $X=[0, \infty)$ and let $d$ be the quasi-metric on $X$ given by $d(x, y)=\max \{y-x, 0\}$ for all $x, y \in X$. It is well known that $(X, d)$ is Smyth complete. Now define $T: X \rightarrow X$ as $T x=0$ if $x \in[0,1]$ and $T x=x / 4$ if $x \in(1, \infty)$. If $x>y>1$ we have $d(T x, T y)=(x-y) / 4$ but $d(x, T x)=d(y, T y)=0$, so that $T$ is not $d$-Kannan on $(X, d)$. However, it is easy to check that $T$ is a Kannan mapping on $\left(X, d^{s}\right)$ for $c=1 / 3$ (note that $d^{s}$ is the Euclidean metric on $X$ ).

2.5. Theorem. Let $(X, d)$ be a d-sequentially complete quasi-metric space. Then, every d-Kannan mapping on $(X, d)$ has a unique fixed point.

Proof. Let $T$ be a $d$-Kannan mapping on $(X, d)$. Then, there exists $c \in[0,1 / 2)$ such that the contraction condition (2.1) follows for all $x, y \in X$. Fix $x_{0} \in X$. From Lemma $2.3(\mathrm{c}),\left(T^{n} x_{0}\right)_{n \in \mathbb{N}}$ is a Cauchy sequence in the metric space $\left(X, d^{s}\right)$. Since $(X, d)$ is $d$-sequentially complete, there exists $z \in X$ such that $\left(T^{n} x_{0}\right)_{n \in \mathbb{N}}$ converges to $z$ for $\tau_{d}$, i.e., $d\left(z, T^{n} x_{0}\right) \rightarrow 0$ as $n \rightarrow \infty$.

Next we show that $T z$ is the unique fixed point of $T$. To this end, we first show that $d(z, T z)=0$. Indeed, we have

$$
\begin{aligned}
d(z, T z) & \leq d\left(z, T^{n} x_{0}\right)+d\left(T^{n} x_{0}, T z\right) \\
& \leq d\left(z, T^{n} x_{0}\right)+c\left(d\left(T^{n-1} x_{0}, T^{n} x_{0}\right)+d(z, T z)\right),
\end{aligned}
$$

for all $n \in \mathbb{N}$. Since $d\left(z, T^{n} x_{0}\right) \rightarrow 0$ and $\left(T^{n} x_{0}\right)_{n \in \mathbb{N}}$ is a Cauchy sequence in the metric space $\left(X, d^{s}\right)$, we deduce that $d(z, T z) \leq c d(z, T z)$. Consequently, $d(z, T z)=0$.

Since by Lemma $2.3(\mathrm{a})$,

$$
d^{s}\left(T z, T^{2} z\right) \leq c\left(d(z, T z)+d\left(T z, T^{2}\right)\right)
$$

we deduce that $d^{s}\left(T z, T^{2} z\right) \leq c d\left(T z, T^{2} z\right)$, so $d^{s}\left(T z, T^{2} z\right)=0$, i.e., $T z$ is a fixed point of $T$.

Finally, if $T u=u$, it follows from Lemma 2.3 (a) that

$$
d^{s}(u, T z)=d^{s}\left(T u, T^{2} z\right) \leq c\left(d(u, T u)+d\left(T z, T^{2} z\right)\right) .
$$

Since $d(u, T u)=d\left(T z, T^{2} z\right)=0$, we deduce that $d^{s}(u, T z)=0$, i.e., $u=T z$. This concludes the proof.

The following examples illustrate Theorem 2.5.

2.6. Example. Let $X=[0, \infty)$ and let $d$ be the quasi-metric on $X$ given by $d(x, y)=\max \{x-y, 0\}$ for all $x, y \in X$. Since $d^{s}$ is the Euclidean metric on $X$, $(X, d)$ is $d$-sequentially complete (in fact, it is left $\mathrm{K}$-sequentially complete because every sequence in $X$ converges to 0 for $\tau_{d}$ ). Define $T: X \rightarrow X$ as in Example 2.4. Let $x, y \in X$, and assume, without loss of generality, that $x \leq y$. If $x, y \in[0,1]$, then $d^{s}(T x, T y)=0$. If $x \in[0,1]$ and $y \in(1, \infty)$ we obtain

$$
d^{s}(T x, T y)=\frac{y}{4} \leq \frac{1}{3}\left(x+\frac{3 y}{4}\right)=\frac{1}{3}(d(x, T x)+d(y, T y)) .
$$


Finally, if $x, y \in(1, \infty)$ we obtain

$$
d^{s}(T x, T y)=\frac{y-x}{4}<\frac{1}{3}\left(\frac{3 x}{4}+\frac{3 y}{4}\right)=\frac{1}{3}(d(x, T x)+d(y, T y)) .
$$

Therefore $T$ is a $d$-Kannan mapping on $(X, d)$ for $c=1 / 3$. Thus, all conditions of Theorem 2.5 are satisfied. In fact $z=0$ is the unique fixed point of $T$.

2.7. Example. Let $X=[0,1] \cup\{2\}$ and let $d$ be the quasi-metric on $X$ given by $d(2, x)=0$ for all $x \in X$, nd $d(x, y)=|x-y|$ otherwise. Clearly $(X, d)$ is d-sequentially complete. Define $T: X \rightarrow X$ as $T 2=0$ and $T x=x / 4$ if $x \in[0,1]$. It is easy to check that $T$ is a d-Kannan mapping on $(X, d)$ for $c=1 / 3$. Thus, all condition of Theorem 2.5 are satisfied. It is interesting to observe that for any $x_{0} \in X$ the sequence $\left(T^{n} x_{0}\right)_{n \in \mathbb{N}}$ converges to 2 for $\tau_{d}$ but 2 is not the fixed point of $T$. This situation illustrates the proof of Theorem 2.5 which shows that $T 2$ is the unique fixed point of $T$; in fact $\left(T^{n} x_{0}\right)_{n \in \mathbb{N}}$ converges to $T 2$ for $\tau_{d^{s}}$.

2.8. Theorem. A quasi-metric space $(X, d)$ is d-sequentially complete if and only if every d-Kannan mapping on $(X, d)$ has a fixed point.

Proof. Suppose that $(X, d)$ is $d$-sequentially complete. Then, every $d$-Kannan mapping on $(X, d)$ has a (unique) fixed point by Theorem 2.5 .

For the converse suppose that $(X, d)$ is not $d$-sequentially complete. Then there exists a Cauchy sequence $\left(x_{n}\right)_{n \in \mathbb{N}}$ in $\left(X, d^{s}\right)$ that does not converge for $\tau_{d}$. Then, for each $x \in X$ there exists $n_{x} \in \mathbb{N}$ such that $d\left(x, x_{n}\right)>0$, for all $n \geq n_{x}$ (indeed, otherwise there is $x \in X$ such that for each $n \in \mathbb{N}$ we can find $m_{n} \geq n$ for which $d\left(x, x_{m_{n}}\right)=0$; since $\left(x_{n}\right)_{n \in \mathbb{N}}$ is a Cauchy sequence in $\left(X, d^{s}\right)$ it follows that $\left(x_{n}\right)_{n \in \mathbb{N}}$ converges to $x$ for $\tau_{d}$, a contradiction).

Now, for each $x \in X$ put $C_{x}=\left\{x_{n}: n \geq n_{x}\right\}$. Clearly $d\left(x, C_{x}\right)>0$ (indeed, if $d\left(x, C_{x}\right)=0$, for some $x \in X$, reasoning as in the parenthetical part of the preceding paragraph, we deduce that that sequence $\left(x_{n}\right)_{n \in \mathbb{N}}$ converges to $x$ for $\tau_{d}$, a contradiction).

Since $\left(x_{n}\right)_{n \in \mathbb{N}}$ is a Cauchy sequence in $\left(X, d^{s}\right)$, for each $x \in X$ there exists $n(x) \geq n_{x}$ such that

$$
d^{s}\left(x_{n}, x_{m}\right)<\frac{1}{4} d\left(x, C_{x}\right)
$$

for all $m, n \geq n(x)$.

Define $T: X \rightarrow X$ as $T x=x_{n(x)}$ for all $x \in X$.

Since $n(x) \geq n_{x}$, we have that $d\left(x, x_{n(x)}\right)>0$, and hence $T$ has not fixed point.

We shall show that, nevertheless, $T$ is a $d$-Kannan mapping on $(X, d)$ for $c=$ $1 / 4$. Indeed, let $x, y \in X$ and suppose, without loss of generality, that $n(x) \leq n(y)$. Then

$$
\begin{aligned}
& d^{s}(T x, T y)=d^{s}\left(x_{n(x)}, x_{n(y)}\right)<\frac{1}{4} d\left(x, C_{x}\right) \\
& \leq \frac{1}{4} d\left(x, x_{n(x)}\right)=\frac{1}{4} d(x, T x) .
\end{aligned}
$$


Since $d(T x, T y) \leq d^{s}(T x, T y)$ and $d(T y . T x) \leq d^{s}(T x, T y)$, we conclude that $T$ is a $d$-Kannan mapping on $(X, d)$ for $c=1 / 4$. This contradiction finishes the proof.

Let $(X, d)$ be a quasi-metric space. The closure for $\tau_{d}$ of a subset $A$ of $X$ will be denoted by $\bar{A}$, and the set of all non-empty closed subsets of the topological $\left(X, \tau_{d}\right)$ by $C l_{d}(X)$.

2.9. Definition. Let $(X, d)$ be a quasi-metric space. By a left-Kannan multivalued mapping on $(X, d)$ we mean a multivalued mapping $T: X \rightarrow C l_{d}(X)$ such that there exists a constant $c \in[0,1 / 2)$ for which the following condition is satisfied:

For each $x, y \in X$ and each $u \in T x$ there exists $v \in T y$ such that

$$
d(u, v) \leq c(d(x, u)+d(y, v)) .
$$

2.10. Theorem. Let $(X, d)$ be a left K-sequentially complete quasi-metric space. Then, every left-Kannan multivalued mapping on $(X, d)$ has a fixed point, i.e., there is $z \in X$ such that $z \in T z$.

Proof. Let $T$ be a left-Kannan multivalued mapping on $(X, d)$. Then, there exists $c \in[0,1 / 2)$ such that the contraction condition (2.2) in Definition 2.9 follows for all $x, y \in X$.

Fix $x_{0} \in X$. Choose $x_{1} \in T x_{0}$. Then, there exists $x_{2} \in T x_{1}$ such that

$$
d\left(x_{1}, x_{2}\right) \leq c\left(d\left(x_{0}, x_{1}\right)+d\left(x_{1}, x_{2}\right)\right)
$$

Therefore

$$
d\left(x_{1}, x_{2}\right) \leq \frac{c}{1-c} d\left(x_{0}, x_{1}\right) .
$$

Following this process we construct a sequence $\left(x_{n}\right)_{n \in \mathbb{N}}$ where $x_{n} \in T x_{n-1}$ and

$$
d\left(x_{n}, x_{n+1}\right) \leq \frac{c}{1-c} d\left(x_{n}, x_{n-1}\right),
$$

for all $n \in \mathbb{N}$. Hence

$$
d\left(x_{n}, x_{n+1}\right) \leq\left(\frac{c}{1-c}\right)^{n} d\left(x_{0}, x_{1}\right)
$$

for all $n \in \mathbb{N}$. Consequently $\left(x_{n}\right)_{n \in \mathbb{N}}$ is a left K-Cauchy sequence in $(X, d)[8$, Proposition 1.2.6]

Since $(X, d)$ is left $\mathrm{K}$-sequentially complete there exists $z \in X$ such that $d\left(z, x_{n}\right) \rightarrow$ 0 as $n \rightarrow \infty$. We shall show that $z \in T z$. Indeed, for each $n \in \mathbb{N}$ there exists $z_{n} \in T z$ such that

$$
d\left(x_{n+1}, z_{n}\right) \leq c\left(d\left(x_{n}, x_{n+1}\right)+d\left(z, z_{n}\right)\right) .
$$

From the triangle inequality and (5) it follows that

$$
d\left(z, z_{n}\right) \leq d\left(z, x_{n+1}\right)+c\left(d\left(x_{n}, x_{n+1}\right)+d\left(z, z_{n}\right)\right),
$$


for all $n \in \mathbb{N}$. Since $d\left(z, x_{n+1}\right) \rightarrow 0$ and $d\left(x_{n}, x_{n+1}\right) \rightarrow 0$ as $n \rightarrow \infty$, we deduce that $d\left(z, z_{n}\right) \rightarrow 0$ as $n \rightarrow \infty$, so $z \in T z$ because $T z$ is closed for $\tau_{d}$. This concludes the proof.

2.11. Lemma (see [8, Proposition 1.2.4]). Let $(X, d)$ be a quasi-metric space. If a left K-Cauchy sequence in $(X, d)$ has a subsequence that converges for $\tau_{d}$ to some $x \in X$, then the sequence converges to $x \in X$ for $\tau_{d}$.

2.12. Theorem. A quasi-metric space $(X, d)$ is left $K$-sequentially complete if and only if every left-Kannan multivalued mapping on $(X, d)$ has a fixed point.

Proof. Suppose that $(X, d)$ is left K-sequentially complete. Then, every leftKannan multivalued mapping on $(X, d)$ has a fixed point by Theorem 2.10.

For the converse suppose that $(X, d)$ is not left K-sequantially complete. Then there exists a left K-Cauchy sequence $\left(x_{n}\right)_{n \in \mathbb{N}}$ in $(X, d)$ that does not converge for $\tau_{d}$. Similarly to the proof of Theorem 2.8, and using Lemma 2.11, we deduce that for each $x \in X$ there exists $n_{x} \in \mathbb{N}$ such that $d\left(x, x_{n}\right)>0$, for all $n \geq n_{x}$.

Now, for each $x \in X$ put $C_{x}=\left\{x_{n}: n \geq n_{x}\right\}$. Then $x \notin \overline{C_{x}}$ and thus $d\left(x, \overline{C_{x}}\right)>0$, where, as usual, $d\left(x, \overline{C_{x}}\right):=\inf \left\{d(x, y): y \in \overline{C_{x}}\right\}$.

Since $\left(x_{n}\right)_{n \in \mathbb{N}}$ is a left $\mathrm{K}$-Cauchy sequence in $(X, d)$, for each $x \in X$ there exists $n(x) \geq n_{x}$ such that

$$
d\left(x_{n}, x_{m}\right)<\frac{1}{4} d\left(x, \overline{C_{x}}\right)
$$

whenever $m \geq n \geq n(x)$.

For each $x \in X$ put $D_{x}=\left\{x_{n}: n \geq n(x)\right\}$. Then $D_{x} \subseteq C_{x}$, so $\overline{D_{x}} \subseteq \overline{C_{x}}$.

Define $T: X \rightarrow C l_{d}(X)$ as $T x=\overline{D_{x}}$ for all $x \in X$.

Since, for each $x \in X, x \notin \overline{C_{x}}$ it follows that $x \notin T x$, and thus $T$ has no fixed points.

We shall show that, nevertheless, $T$ is a left-Kannan multivalued mapping on $(X, d)$ for $c=1 / 3$. Indeed, let $x, y \in X$ and suppose, without loss of generality that $n(x) \leq n(y)$. Then $D_{y} \subseteq D_{x}$, so $T y \subseteq T x$, and hence for each $u \in T y$ we can take $v=u \in T x$, and thus $d(u, v)=0$. On the other hand, given $u \in T x$ there exists $v \in T y$ such that $d(u, v)<d\left(x, \overline{C_{x}}\right) / 12+d(u, T y)$. Since for each $\varepsilon>0$ there exists $n_{\varepsilon} \geq n(x)$ such that $d\left(u, x_{n_{\varepsilon}}\right)<\varepsilon$ we deduce (recall that $x_{n(y)} \in T y$ and $\left.T_{x} \subseteq \overline{C_{x}}\right)$ :

$$
\begin{aligned}
d(u, v) & <\frac{1}{12} d\left(x, \overline{C_{x}}\right)+d(u, T y) \leq \frac{1}{12} d\left(x, \overline{C_{x}}\right)+d\left(u, x_{n_{\varepsilon}}\right)+d\left(x_{n_{\varepsilon}}, T y\right) \\
& <\frac{1}{12} d\left(x, \overline{C_{x}}\right)+\varepsilon+d\left(x_{n_{\varepsilon}}, x_{n(y)}\right)<\frac{1}{12} d\left(x, \overline{C_{x}}\right)+\varepsilon+\frac{1}{4} d\left(x, \overline{C_{x}}\right) \\
& \leq \varepsilon+\frac{1}{3} d\left(x, T_{x}\right) \leq \varepsilon+\frac{1}{3} d(x, u) .
\end{aligned}
$$

Since $\varepsilon$ is arbitrary we deduce that

$$
d(u, v) \leq \frac{1}{3} d(x, u) .
$$


We have shown that $T$ is a left-Kannan multivalued mapping on $(X, d)$ for $c=1 / 3$. This finishes the proof.

2.13. Remark. Let $(\mathbb{R}, d)$ be the quasi-metric space of Example 1.6. By Theorem 2.5 , every $d$-Kannan mapping on $(\mathbb{R}, d)$ has a unique fixed point. However there exists a left-Kannan multivalued mapping on it without fixed points, by Theorem 2.12. Finally, if $(X, d)$ is the quasi-metric space of Example 2.4 or the quasi-metric space of Example 2.6, then every left-Kannan multivalued mapping on $(X, d)$ has a fixed point by Theorem 2.12.

Acknowledgement. Carmen Alegre, Salvador Romaguera and Pedro Tirado are supported under grant MTM2015-64373-P (MINECO/FEDER, UE).

\section{References}

[1] C. Alegre and J. Marín, Modified w-distances on quasi-metric spaces and a fixed point theorem on complete quasi-metric spaces, Top. Appl. 203 (2016), 32-41.

[2] C. Alegre, J. Marín and S. Romaguera, A fixed point theorem for generalized contractions involving $w$-distances on complete quasi-metric spaces, Fixed Point Theory Appl. 2014, 2014:40.

[3] S. Al-Homidan, Q.H. Ansari and J.C. Yao, Some generalizations of Ekeland-type variational principle with applications to equilibrium problems and fixed point theory, Nonlinear Anal. TM\&A 69 (2008), 126-139.

[4] M. Ali-Akbari, B. Honari, M. Pourmahdian and M.M. Rezaii, The space of formal balls and models of quasi-metric spaces, Math. Struct. Comput. Sci. 19 (2009), 337-355.

[5] I. Altun, N. Al Arifi, M. Jleli, A. Lashin and B. Samet, A new concept of $\left(\alpha, F_{d}\right)$-contraction on quasi metric space, J. Nonlinear Sci. Appl. 9 (2016), 3354-3361.

[6] J. Caristi, Fixed point theorems for mappings satisfying inwardness conditions, Trans. Amer. Math. Soc. 215 (1976), 241-251.

[7] S. Cobzaş, Completeness in quasi-metric spaces and Ekeland Variational Principle, Top. Appl. 158 (2011), 1073-1084.

[8] S. Cobzaş, Functional Analysis in Asymmetric Normed Spaces, Birkhäuser, Springer Basel, 2013.

[9] H. Dağ, G. Minak and I. Altun, Some fixed point results for multivalued $F$-contractions on quasi metric spaces, RACSAM, DOI: 10.1007/s13398-016-0285-3, to appear.

[10] T.K. Hu, On a fixed point theorem for metric spaces, Amer. Math. Monthly 74 (1967), 436-437.

[11] R. Kannan, Some results on fixed points, Bull. Calcutta Math. Soc. 60 (1968), 71-76.

[12] E. Karapinar and S. Romaguera, On the weak form of Ekeland's Variational Principle in quasi-metric spaces, Top. Appl. 184 (2015), 54-60.

[13] A.W. Kirk, Caristi's fixed point theorem and metric convexity, Colloq. Math. 36 (1976), 81-86.

[14] H.P.A. Künzi, Nonsymmetric distances and their associated topologies: About the origins of basic ideas in the area of asymmetric topology, in: C.E. Aull, R. Lowen (Eds.), Handbook of the History of General Topology, vol. 3, Kluwer, Dordrecht, 2001, pp. 853-968.

[15] A. Latif, and S.A. Al-Mezel, Fixed point results in quasimetric spaces, Fixed Point Theory Appl. 2011 (2011), Article ID 178306, 8 pages.

[16] J. Marín, S. Romaguera and P. Tirado, $Q$-functions on quasi-metric spaces and fixed points for multivalued maps, Fixed Point Theory Appl. 2011 (2011), Article ID 603861, 10 pages.

[17] J. Marín, S. Romaguera and P. Tirado, Generalized contractive set-valued maps on complete preordered quasi-metric spaces, J. Funct. Spaces Appl. 2013 (2013), Article ID 269246, 6 pages.

[18] S. Park, Characterizations of metric completeness, Colloquium Mathematicum 49 (1984), 21-26. 
[19] I.L. Reilly, P.V. Subrahmanyam and M.K. Vamanamurthy, Cauchy sequences in quasipseudo-metric spaces, Mh. Math. 93 (1982), 127-140.

[20] S. Romaguera, M.P. Schellekens and O. Valero, Complexity spaces as quantitative domains of computation, Top. Appl. 158 (2011), 853-860.

[21] S. Romaguera and P. Tirado, The complexity probabilistic quasi-metric space, J. Math. Anal. Appl. 376 (2011), 732-740.

[22] S. Romaguera and P Tirado, A characterization of Smyth complete quasi-metric spaces via Caristi's fixed point theorem, Fixed Point Theory Appl. 2015, 2015:183.

[23] S. Romaguera and O. Valero, Domain theoretic characterisations of quasi-metric completeness in terms of formal balls, Math. Struct. Comput. Sci. 20 (2010), 453-472.

[24] M.P. Schellekens, A characterization of partial metrizability: domains are quantifiable, Theor. Comput. Sci. 305 (2003), 409-432.

[25] N. Shioji, T. Suzuki, W. Takahashi, Contractive mappings, Kannan mappings and metric completeness, Proc. Amer. Math. Soc. 126 (1998), 3117-3124.

[26] P.V. Subrahmanyam, Completeness and fixed-points, Mh. Math. 80 (1975), 325-330.

[27] T. Suzuki, W. Takahashi, Fixed point theorems and characterizations of metric completeness, Top. Methods Nonlinear Anal. 8 (1996), 371-382.

[28] T. Suzuki, A generalized Banach contraction principle that characterizes metric completeness, Proc. Amer. Math. Soc., 136 (2008), 1861-1869. 